\title{
A Biologically Motivated Scheme for Robust Junction Detection
}

\author{
Thorsten Hansen and Heiko Neumann
}

\author{
Univ. Ulm, Dept. of Neural Information Processing, D-89069 Ulm, Germany \\ (hansen, hneumann) @neuro. informatik . uni-ulm.de
}

\begin{abstract}
Junctions provide important cues in various perceptual tasks, such as the determination of occlusion relationship for figureground separation, transparency perception, and object recognition, among others. In computer vision, junctions are used in a number of tasks like point matching for image tracking or correspondence analysis. We propose a biologically motivated approach to junction detection. The core component is a model of V1 based on biological mechanisms of colinear long-range integration and recurrent interaction. The model V1 interactions generate a robust, coherent representation of contours. Junctions are then implicitly characterized by high activity for multiple orientations within a cortical hypercolumn. A local measure of circular variance is used to extract junction points from this distributed representation. We show for a number of generic junction configurations and various artificial and natural images that junctions can be accurately and robustly detected. In a first set of simulations, we compare the detected junctions based on recurrent long-range responses to junction responses as obtained for a purely feedforward model of complex cells. We show that localization accuracy and positive correctness is improved by recurrent long-range interaction. In a second set of simulations, we compare the new scheme with two widely used junction detection schemes in computer vision, based on Gaussian curvature and the structure tensor. Receiver operator characteristic (ROC) analysis is used for a threshold-free evaluation of the different approaches. We show for both artificial and natural images that the new approach performs superior to the standard schemes. Overall we propose that nonlocal interactions as realized by long-range interactions within V1 play an important role for the detection of higher order features such as corners and junctions.
\end{abstract}

\section{Introduction and Motivation}

Corners and junctions are points in the image where two or more edges join or intersect. Whereas edges lead to variations of the image intensity along a single direction, corners and junctions are characterized by variations in at least two directions. Compared to regions of homogeneous intensity, edges are rare events. Likewise, compared to edges, corners and junctions are rare events of high information content. Moreover, corners and junctions are invariant under different viewing angles and viewing distances. Both the sparseness of the signal 
and the invariance under affine transformations and scale variations establish corners and junctions as important image features.

Corners and junctions are useful for various higher level vision tasks such as the determination of occlusion relationships, matching of stereo images, object recognition and scene analysis. The importance of corner and junction points for human object recognition has been demonstrated in a number of psychophysical experiments (Attneave, 1954; Biederman, 1985). Junctions also seem to play an important role in the perception of brightness and transparency (Adelson, 2000; Metelli, 1974) and have been proposed to trigger modal and amodal surface completion (Rubin, 2001). In physiological studies in monkey visual cortex cells have been reported which selectively respond to corners and line-ends (Hubel and Wiesel, 1968) as well as curved patterns and angles (Pasupathy and Connor, 2001).

Recently (McDermott, 2001) studied the performance of human observers for the detection of junctions in natural images. He found that the ability to detect junctions is severely impaired if subjects could view the location of a possible junction only through a small aperture. Detection performance and observers' confidence ratings decreased with decreasing size of the aperture. The results suggest that a substantial number of junctions in natural images cannot be detected by local mechanisms.

In this paper we propose a new mechanism for corner and junction detection based on a distributed representation of contour responses within hypercolumns (Zucker et al., 1989). Unlike local approaches as proposed in computer vision (Harris, 1987; Mokhtarian and Suomela, 1998), the new scheme is based on a more global, recurrent long-range interaction for the coherent computation of contour responses. Such nonlocal interactions evaluate local responses within a more global context and generate a robust contour representation. A measure of circular variance is used to extract corner and junctions points at positions of large responses for more than one orientation.

The paper is organized as follows. In Sec. 2 we present the model of recurrent colinear long-range interactions and detail the new junction detection scheme. Simulation results for a number of artificial and natural images are presented in Sec. 3. Section 3 concludes the paper.

\section{A Neural Model for Corner and Junction Detection}

Corner and junction configurations can be characterized by high responses for two or more orientations at a particular point in the visual space. A cortical hypercolumn is the neural representation for orientated responses at a particular point. Corners and junctions are thus characterized by significant activity of multiple neurons within a hypercolumn.

Multiple oriented activities as measured by a simple feedforward mechanism are sensitive to noisy signal variations. In previous work we have proposed a model of recurrent colinear long-range interaction in the primary visual cortex for contour enhancement (Hansen and Neumann, 1999, 2001). During the recur- 
rent long-range interactions, the initially noisy activities are evaluated within a larger context. In this recurrent process, only coherent orientations responses are preserved, i.e., responses which are supported by responses in the spatial neighborhood, while other responses are suppressed. Besides the enhancement of coherent contours, the proposed model also preserves multiple activities at corners and junctions. Corners and junctions are thus implicitely characterized by a distributed representation of high multiple activity within a hypercolumn.

Such a distributed representation may suffice for subsequent neural computations. However, at least for the purpose of visualization and comparison to other junction detection schemes an explicit representation is requested. Following the above considerations, corners and junctions can be marked if multiple orientations are active and high overall activity exists within a hypercolumn.

In the following we first present the proposed model of colinear recurrent longrange interactions in V1 (Sec. 2.1), and then detail a mechanism to explicitely mark corner and junction points (Sec. 2.2).

\subsection{Coherent Contour Representation by a Model of Colinear Recurrent Long-Range Interaction in V1}

The model of colinear long-range interactions in V1 is motivated by a number of biological mechanisms. The core mechanisms of the model include localized receptive fields for oriented contrast processing, interlaminar feedforward and feedback processing, cooperative horizontal long-range integration, and lateral competitive interactions. The key properties of the model are motivated by empirical findings, namely (i) horizontal long-range connections (Gilbert and Wiesel, 1983; Rockland and Lund, 1983) between cells with colinear aligned RFs (Bosking et al., 1997); (ii) inhibitory, orientation-unspecific short-range connections (Bosking et al., 1997); and (iii) modulating feedback which cannot drive a cell but modulates initial bottom-up activity (Hirsch and Gilbert, 1991, Hupé et al., 1998). The model architecture is defined by a sequence of preprocessing stages and a recurrent loop of long-range interaction, realizing a simplified architecture of V1 (Fig. 1).

Feedforward Preprocessing. In the feedforward path, the initial luminance distribution is processed by isotropic LGN-cells, followed by orientation-selective simple and complex cells. The interactions in the feedforward path are governed by basic linear equations to keep the processing in the feedforward path relatively simple and to focus on the contribution of the recurrent interaction. In our model, complex cell responses $C_{\theta}$ (as output of the feedforward path, cf. (Fig. 1) provide an initial local estimate of contour strength, position and orientation which is used as bottom-up input for the recurrent loop. A more detailed description of the computation in the feedforward path can be found in Hansen and Neumann (1999).

Recurrent Long-Range Interaction. The output of the feedforward preprocessing defines the input to the recurrent loop. The recurrent loop has two 


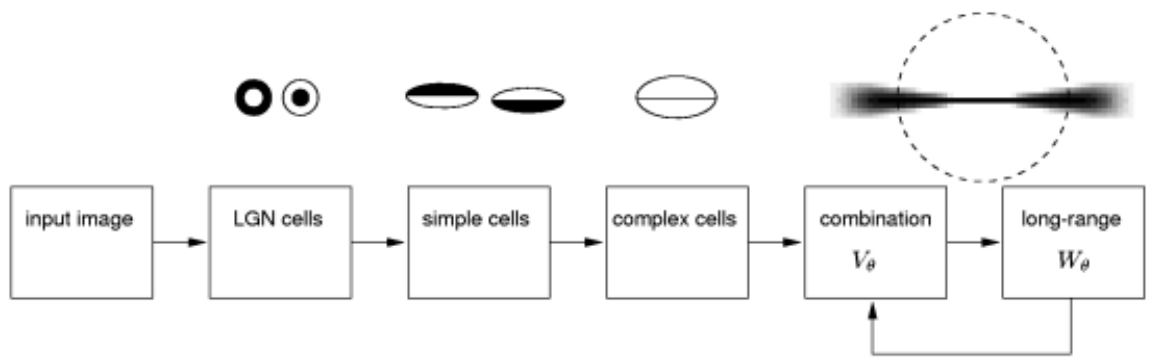

Fig. 1. Overview of model stages together with a sketch of the sample receptive fields of cells at each stage for $0^{\circ}$ orientation. For the long-range stage, the spatial weighting function of the long-range filter is shown together with the spatial extend of the inhibitory short-range interactions dashed circle.

stages, namely a combination stage where bottom-up and top-down inputs are fused, and a stage of long-range interaction.

Combination Stage. At the combination stage, feedforward complex cell responses and feedback long-range responses are combined. Feedforward inputs $C_{\theta}$ and feedback inputs $W_{\theta}$ are added and subject to shunting inhibition

$$
\partial_{t} V_{\theta}=-\alpha_{V} V_{\theta}+\left(\beta_{V}-V_{\theta}\right) \text { net }_{\theta}, \quad \text { where } \text { net }_{\theta}=C_{\theta}+\delta_{V} W_{\theta} .
$$

Solving the equation at equilibrium $\partial_{t} V_{\theta}=0$ results in a normalization of activity

$$
V_{\theta}=\beta_{V} \frac{\operatorname{net}_{\theta}}{\alpha_{V}+\operatorname{net}_{\theta}}
$$

The weighting parameter $\delta_{V}=2$ is chosen so that dimensions of $C_{\theta}$ and $W_{\theta}$ are approximately equal, the decay parameter $\alpha_{V}=0.2$ is chosen small compared to net ${ }_{\theta}$, and $\beta_{V}=10$ scales the activity to be sufficiently large for the subsequent long-range interaction. For the first iteration step, feedback responses $W_{\theta}$ are set to $C_{\theta}$.

Long-Range Interaction. At the long-range stage the contextual influences on cell responses are modeled. Orientation-specific, anisotropic long-range connections provide the excitatory input. The inhibitory input is given by isotropic interactions in both the spatial and orientational domain. Long-range connections are modeled by a filter whose spatial layout is similar to the bipole filter as first proposed by Grossberg and Mingolla (1985). The spatial weighting function of the long-range filter is narrowly tuned to the preferred orientation, reflecting the highly significant anisotropies of long-range fibers in visual cortex (Bosking et al., 1997). The size of the long-range filter is about four times the size of the RF of a complex cell, while the size of the short-range connections is about 2.5 times the size of the complex cell RF, as sketched in Fig. 1. 
Essentially, excitatory input is provided by correlation of the feedforward input with the long-range filter $B_{\theta}$. A cross-orientation inhibition prevents the integration of cells responses at positions where responses for the orthogonal orientation also exist. The excitatory input is governed by

$$
\operatorname{net}_{\theta}^{+}=\left[V_{\theta}-V_{\theta^{\perp}}\right]^{+} \star B_{\theta}
$$

where $\star$ denotes spatial correlation and $[x]^{+}=\max \{x, 0\}$ denotes half-wave rectification.

The profile of the long-range filter is defined by a directional term $D_{\varphi}$ and a proximity term generated by a circle $C_{r}$ of radius $r=25$ which is blurred by an isotropic 2D Gaussian $G_{\sigma}, \sigma=3$. The detailed equations read

$$
\begin{aligned}
& B_{\theta, \alpha, r, \sigma}(x, y)=D_{\varphi} \cdot C_{r} \star G_{\sigma} \\
& D_{\varphi}= \begin{cases}\cos \left(\frac{\pi / 2}{\alpha} \varphi\right) & \text { if }|\varphi|<\alpha \\
0 & \text { otherwise },\end{cases}
\end{aligned}
$$

where $\varphi=\varphi(\theta)$ is defined as atan2 $\left(\left|y_{\theta}\right|,\left|x_{\theta}\right|\right)$ and $\left(x_{\theta}, y_{\theta}\right)^{\mathrm{T}}$ denotes the vector $(x, y)^{\mathrm{T}}$ rotated by $\theta$. The operator - denotes the point-wise multiplication of two filter kernels or $2 \mathrm{D}$ matrices. The parameter $\alpha=10^{\circ}$ defines the opening angle of $2 \alpha$ of the long-range filter. The factor $\frac{\pi / 2}{\alpha}$ maps the angle $\varphi$ in the range $[-\alpha, \alpha]$ to the domain $[-\pi / 2, \pi / 2]$ of the cosine function with positive range. A plot of the long-range filter for a reference orientation of $0^{\circ}$ is depicted in (Fig. 1) (top right inset).

Responses which are not salient in the sense that nearby cells of similar orientation preference also show strong activity should be diminished. Thus an inhibitory term is introduced which samples activity from both orientational $\widetilde{g}_{\sigma_{o}, \theta}$, $\sigma_{o}=0.5$, and spatial neighborhood $G_{\sigma_{\text {sur }}}, \sigma_{\text {sur }}=8$ :

$$
\operatorname{net}_{\theta}^{-}=\operatorname{net}_{\theta}^{+} \circledast \widetilde{g}_{\sigma_{o}, \theta} \star G_{\sigma_{\text {sur }}}
$$

where $\circledast$ denotes correlation in the orientation domain. The orientational weighting function $\widetilde{g}_{\sigma_{o}, \theta}$ is implemented by a $1 \mathrm{D}$ Gaussian $g_{\sigma_{o}}$, discretized on a zero-centered grid of size $o_{\max }$, normalized, and circularly shifted so that the maximum value is at the position corresponding to $\theta$. The spatially inhibitory interactions $G_{\sigma_{\text {sur }}}$ model the short-range connections.

Excitatory and inhibitory terms combine through shunting interaction

$$
\partial_{t} W_{\theta}=-\alpha_{W} W_{\theta}+\beta_{W} V_{\theta}\left(1+\eta^{+} \operatorname{net}_{\theta}^{+}\right)-\eta^{-} W_{\theta} \operatorname{net}_{\theta}^{-} .
$$

The equation is solved at equilibrium, resulting in a nonlinear, divisive interaction

$$
W_{\theta}=\beta_{W} \frac{V_{\theta}\left(1+\eta^{+} \operatorname{net}_{\theta}^{+}\right)}{\alpha_{W}+\eta^{-} \operatorname{net}_{\theta}^{-}} .
$$

where $\alpha_{W}=0.2$ is the decay parameter and $\eta^{+}=5, \eta^{-}=2$, and $\beta_{W}=0.001$ are scale factors. 
Activity $W_{\theta}$ from long-range integration is gated by the activity $V_{\theta}$ and thus implements a modulating rather than generating effect of lateral interaction on cell activities (Hirsch and Gilbert, 1991; Hué et al., 1998). The result of the long-range stage is fed back and combined with the feedforward complex cell responses, thus closing the recurrent loop. The shunting interactions governing both the long-range interactions and the combination of feedback and feedforward input ensure rapid equilibration of the dynamics after a few recurrent cycles resulting in graded responses within a bounded range of activations ("analog sensitivity", Grossberg et al., 1997).

The model is robust against parameter changes which is mainly caused by the compressive transformation equations employed. For the combination of responses (Eq. 2), however, it is crucial to have activities in both streams of a similar order of magnitude. Also the relative RF sizes must not be substantially altered.

\subsection{Junction Detection by Read-Out of Distributed Information Using a Measure of Circular Variance}

As stated above, corners and junction are characterized by points in the visual space where responses for multiple orientations are present and high overall activity exists within a hypercolumn. For the read-out of this distributed information a measure of circular variance is used to signal multiple orientation. The overall activity is given by the sum across all orientation within a hypercolumn. Thus, the junction map $J$ for a distributed hypercolumnar representation such as the activity of the long-range stage $W_{\theta}$ (Eq. 8) is given by

$$
J=\operatorname{circvar}(W)^{2} \sum_{\theta} W_{\theta}, \text { where } \operatorname{circvar}(W)=1-\left|\sum_{\theta} W_{\theta} \exp (2 \mathrm{i} \theta)\right| / \sum_{\theta} W_{\theta} .
$$

The function "circvar" is a measure of the circular variance within a hypercolumn. The squaring operation enhances the response if the circular variance assumes high values. Circular variance takes values in the range [0,1]. A circular variance of 0 denotes a single response, whereas a value of 1 occurs if all orientations have the same activity.

To precisely localize the junction points, the junction map $J$ is first smoothed with a Gaussian $G_{\sigma}, \sigma=3$. Junction points are then marked as local maxima whose strength must exceed a fraction $\kappa=0.25$ of the maximum response in the smoothed junction map. Local maxima are computed within a $3 \times 3$ neighborhood.

\section{Simulation and Evaluation}

In this section we show the competencies of the proposed junction detection scheme for a variety of synthetic and natural images. In particular, the localization properties of the new scheme and the detection performance on natural 
images are evaluated. In the first part of this section, we compare the detected junctions based on recurrent long-range responses to junction responses as obtained for a purely feedforward model of complex cells to demonstrate the advantages of the recurrent long-range interaction. In the second part, we compare the new scheme with two junction detection schemes widely used in computer vision, based on Gaussian curvature and the structure tensor. Receiver operator characteristic (ROC) analysis (Green and Swets, 1974) is used for a thresholdfree evaluation of the different approaches (Hansen, 2002). Model parameters as specified in Sec. 2 are used in all simulations, and 12 recurrent cycles were computed to generate the long-range responses.

\subsection{Evaluation of Junction Detection Based on Feedforward vs. Recurrent Long-Range Processing}

In order to focus on the relative merits of the recurrent long-range interactions for the task of corner and junction detection, the proposed scheme is evaluated using two different kinds of input, namely the activity $W_{\theta}$ of the long-range stage and the purely feedforward activity $C_{\theta}$ of the complex cell stage.

Localization of Generic Junction Configurations. From the outset of corner and junction detection in computer vision, the variety of junction types has been partitioned into distinct classes like T-, L-, and W-junctions, (Huffman, 1971), and more recently, $\Psi$-junctions (Adelson, 2000). In the first simulation we compare the localization accuracy of junction responses based on feedforward vs. recurrent long-range responses for L-, T-, Y-, W- and $\Psi$-junctions (Fig. 2). For all junction types, the localization is considerably better for the method based on the recurrent long-range interaction.

Processing of Images. We have also evaluated the junction detection performance on real world images, such as cubes within a laboratory environment (Fig. 3). At the complex cell stage, many false responses are detected due to noisy variations of the initial orientation measurement. These variations are reduced at the long-range stage by the recurrent interaction, such that only the positions of significant orientation variations remain. We have further employed ROC analysis for the threshold-free evaluation of the detection performance. The results show a better performance of the recurrent approach over the full range of thresholds (Fig. 3, right).

\subsection{Evaluation of Detection Performance Compared to Other Junction Detection Schemes}

In this section we compare the new scheme based on recurrent long-range interaction with two junction detection schemes proposed in computer vision that utilize only localized neighborhoods, namely the structure tensor (Förstner, 1986; Harris, 1987; Nitzberg and Shiota, 1992) and Gaussian curvature (Beaudet, 1978; 


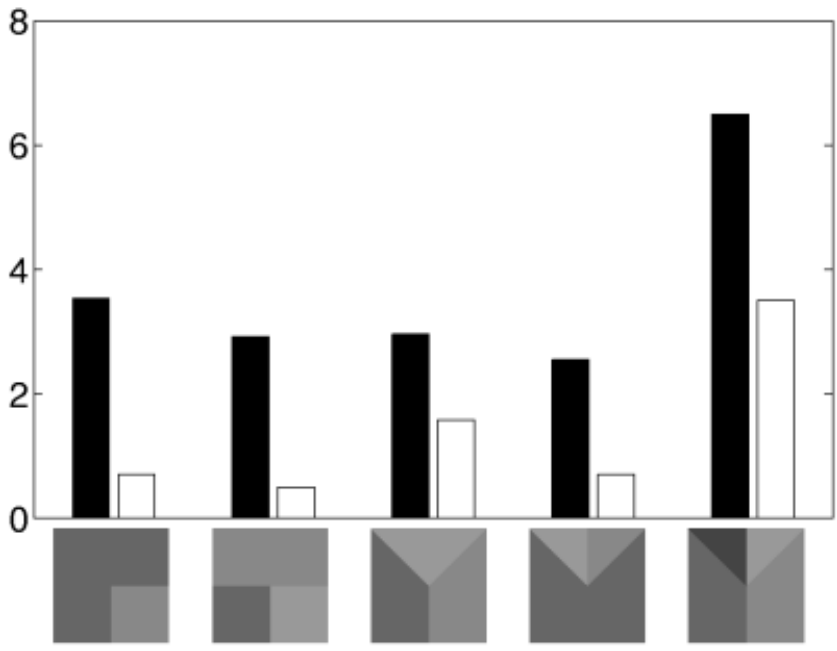

Fig. 2. Distance in pixels from ground-truth location ordinate for L-, T-, Y-, W- and $\Psi$-junctions abscissa. Deviation from ground-truth position is considerably smaller for the recurrent long-range interaction open bars compared to the complex cell responses solid bars.
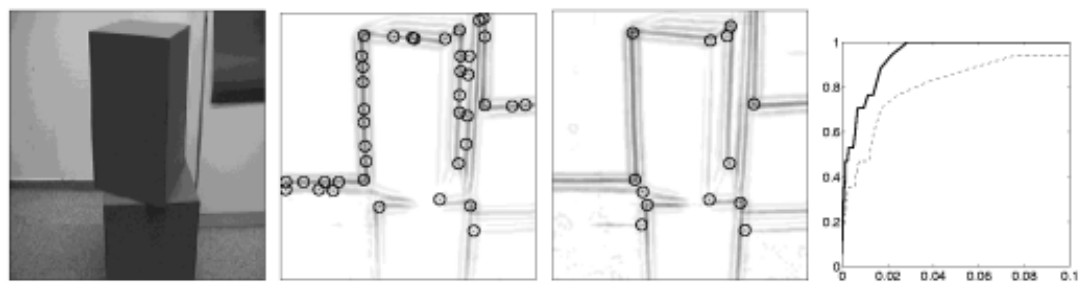

Fig. 3. Simulation of the junction detection scheme for cube images in a laboratory environment. The size of the images is $230 \times 246$ pixels. Left to right: Input image; detected corners and junctions based on the complex cell responses (CX); based on the long-range responses (LR); and the corresponding ROC curves (solid lines LR; dashed lines $\mathrm{CX}$ ). For better visualization, a cut-out of the left part of the ROC curves is shown. The recurrent long-range interaction results in a decrease of circular variance along object contours and thus eliminates a number of false positive responses.

Zetzsche and Barth, 1990). Both schemes compute the first- or second-order derivatives of the image intensity values, respectively. For a fair comparison of methods one has to ensure that all junction detectors operate on (at least approximately) the same scale (Lindeberg, 1998). The derivatives used in the two standard methods are therefore approximated by Gaussian derivatives whose standard deviations are parameterized to fit the successive convolution of filter masks used to compute the complex cell responses. We show the results of the 
ROC analysis when applied to a number of artificial and natural images, particularly a series of cube images within a laboratory environment (Fig. 3), and a second set of images containing an artificial corner test image from Smith and Brady (1997), a laboratory scene from Mokhtarian and Suomela (1998) and an image of a staircase (Fig. 5). For all images, the ROC curve for the new scheme based on recurrent long-range interactions is well above the ROC curves for the other schemes, indicating a higher accuracy of the new method.
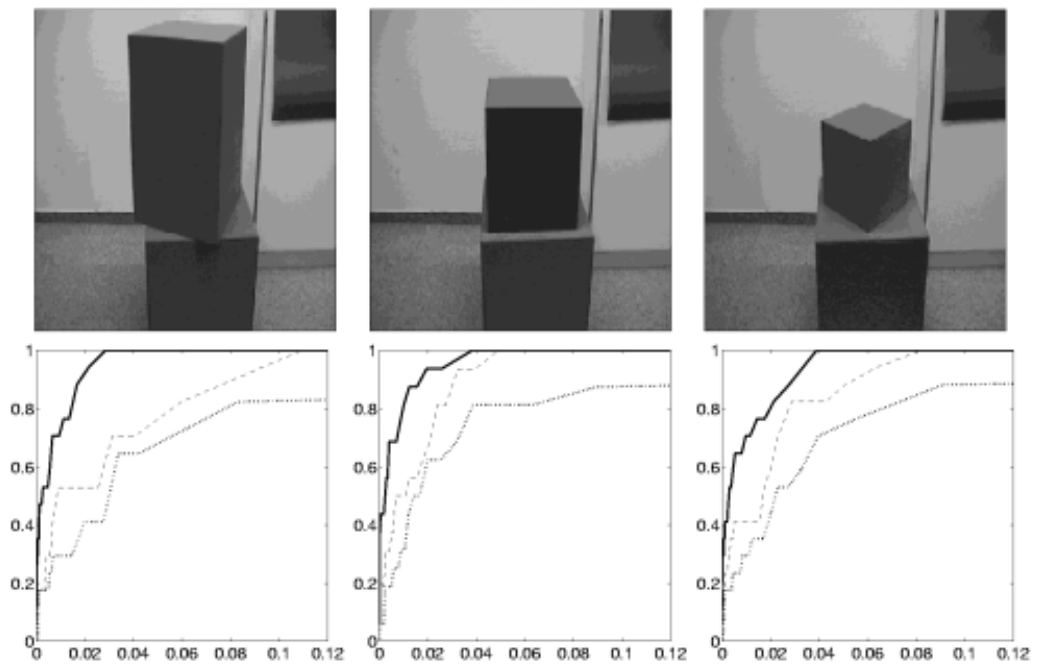

Fig. 4. Top row: Cube images in a laboratory environment. Bottom: ROC analysis of junction detection performance of the new scheme solid compared to other schemes based on Gaussian curvature dashed, and on the structure tensor dotted. For better visualization, a cut-out of the left part of the ROC curves is shown.

\section{Conclusion}

We have proposed a novel mechanism for corner and junction detection based on a distributed representation of orientation information within a cortical hypercolumn. The explicit representation of a number of orientations in a cortical hypercolumn is shown to constitute a powerful and flexible, multipurpose scheme which can be used to code intrinsically $1 \mathrm{D}$ signal variations like contours as well as $2 \mathrm{D}$ variations like corners and junctions. Orientation responses within a hypercolumn can be robustly and reliably computed by using contextual information. We have proposed a model of recurrent long-range interactions to compute coherent orientation responses. In the context of corner and junction detection we have demonstrated the benefits of using contextual information and recurrent interactions, leading to a considerable increase in localization accuracy and 

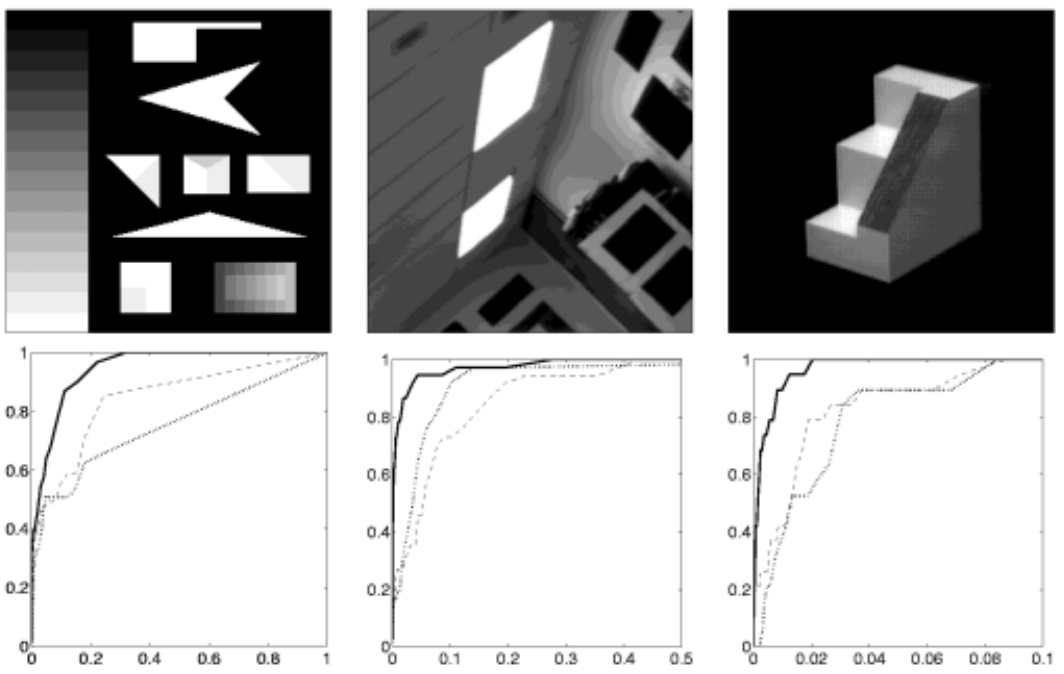

Fig. 5. Top: Input images. Bottom: ROC analysis of junction detection performance of the new scheme solid compared to other schemes based on Gaussian curvature dashed, and on the structure tensor dotted. For better visualization, a cut-out of the left part of the ROC curves is shown.

detection performance compared to a simple feedforward scheme and to local methods as proposed in computer vision. In the context of neural computation we have shown that junctions can be robustly and reliably represented by a suggested biological mechanism based on a distributed hypercolumnar representation and recurrent colinear long-range interactions.

\section{References}

Adelson, E. H. (200) Lightness perception and lightness illusions. In Gazzaniga, M. S., ed. The New Cognitive Neurosciences, pp. 339-351. MIT Press, Cambridge, MA, 2 edn.

Attneave, F. (1954) Some informational aspects of visual perception. Psychol. Rev., 61(3):183-193.

Beaudet, P.R. (1978) Rotationally invariant image operators. In 4th Int. Joint Conf. on Pattern Recognition, pp. 578-583, Kyoto, Japan.

Biederman, I. (1985) Human image understanding: recent research and a theory. Computer Vision, Graphics, Image Proc., 32(1):29-73.

Bosking, W. H., Zhang, Y., Schofield, B., Fitzpatrick, D. (1997) Orientation selectivity and the arrangement of horizontal connections in tree shrew striate cortex. J. Neurosci., 17(6):2112-2127.

Förstner, W. (1986) A feature based correspondence algorithm for image matching. In Int. Arch. Photogramm. Remote Sensing, volume 26, pp. 176-189.

Gilbert, C. D. and Wiesel, T. N. (1983) Clustered intrinsic connections in cat visual cortex. J. Neurosci., 3:1116-1133. 
Green, D. M. and Swets, J. A. (1974) Signal Detection Theory and Psychophysics. Krieger, Huntington, NY, 1974.

Grossberg, S. and Mingolla, E. (1985) Neural dynamics of form perception: boundary completion, illusory figures, and neon color spreading. Psychol. Rev., 92:173-211, 1985.

Hansen, T. (2002) A neural model of early vision: contrast, contours, corners and surfaces. Doctoral dissertation, Univ. Ulm, Faculty of Computer Science, Dept. of Neural Information Processing. Submitted.

Hansen, T. and Neumann, H. (1999) A model of V1 visual contrast processing utilizing long-range connections and recurrent interactions. In Proc. 9. Int. Conf. on Artificial Neural Networks (ICANN99), pp. 61-66, Edinburgh, UK.

Hansen, T. and Neumann, H. (2001) Neural mechanisms for representing surface and contour features. In Stefan Wermter, Jim Austin, and David Willshaw, editors, Emergent Neural Computational Architectures Based on Neuroscience, LNCS/LNAI 2036, pp. 139-153. Springer, Berlin Heidelberg.

Harris, C. J. (1987) Determination of ego-motion from matched points. In Proc. Alvey Vision Conference, pp. 189-192, Cambridge, UK.

Hirsch, J.A. and Gilbert, C.D. (1991) Synaptic physiology of horizontal connections in the cat's visual cortex. J. Neurosci., 11(6):1800-1809.

Hubel,D. H. and Wiesel, T. N. (1968) Receptive fields and functional architecture of monkey striate cortex. J. Physiol., 195:215-243.

Huffman, D. A. (1971) Impossible objects as nonsense sentences. In B. Meltzer and D. Michic, editors, Machine Intelligence 6, pp. 295-323. Edinburgh University Press, Edinburgh.

Hupé, J.M., James, A.C., Payne, B.R., Lomber, S.G., Girard, P. and Bullier, J.(1998) Cortical feedback improves discrimination between figure and background by V1, V2 and V3 neurons. Nature, 394:784-787.

Lindeberg, T. (1998) Feature detection with automatic scale selection. Int. J. Comput. Vision, 30(2):77-116.

McDermott, J.H. (2001) Some experiments on junctions in real images. Master's thesis, University Colledge London, 2001. Online available from http://persci.mti.edu/ ${ }^{j m c d e r m . ~}$

Metelli, F. (1974) The perception of transparency. Sci. Am., 230(4):90-98.

Mokhtarian, F. and Suomela, R. (1998) Robust image corner detection through curvature scale space. IEEE Trans. Pattern Anal. Mach. Intell., 20(12):1376-1381.

Nitzberg, M. and Shiota, T. (1992) Nonlinear image filtering with edge and corner enhancement. IEEE Trans. Pattern Anal. Mach. Intell., 14:826-833.

Pasupathy, A. and Connor, C. E. (2001) Shape representation in area V4: positionspecific tuning for boundary conformation. J. Neurophysiol., 86(5):2505-2519.

Rockland, K. S. and Lund, J. S. (1983) Intrinsic laminar lattice connections in primate visual cortex. J. Comp. Neurol., 216:303-318.

Rubin, N. (2001) The role of junctions in surface completion and contour matching. Perception, 30(3):339-366.

Smith, S. and Brady, J. (1997) SUSAN - a new approach to low level image processing. Int. J. Comput. Vision, 23(1):45-78.

Zetzsche, C. and Barth, E. (1990) Fundamental limits of linear filters in the visual processing of two-dimensional signals. Vision Res., 30(7):1111-1117.

Zucker, S. W., Dobbins, A. and Iverson, L. A. (1989) Two stages of curve detection suggest two styles of visual computation. Neural Comput., 1:68-81. 